\title{
Influence of Pulse Heating on Rayleigh Area of Amorphous FINEMET-Type Alloy
}

\author{
L. NOVÁK ${ }^{a}$, J. KOVÁČ ${ }^{b, *}$ AND L. HuBAČ $\check{C}^{a}$ \\ ${ }^{a}$ Department of Physics, Technical University of Košice, Park Komenského 2, 04200 Košice, Slovakia \\ ${ }^{b}$ Institute of Experimental Physics, SAS, Watsonova 47, 04001 Košice, Slovakia
}

\begin{abstract}
The effect of pulse annealing on magnetization processes in FINEMET-type alloy in weak magnetic fields (the Rayleigh area) is studied in this paper. Rapidly quenched FINEMET ribbon in the original amorphous state was chosen for the study. The significant differences in behavior of studied materials are ascribed to the differences in internal mechanical stresses, which bring about the changes in total magnetic anisotropy and consequently also changes in domain walls thickness.
\end{abstract}

DOI: 10.12693/APhysPolA.131.753

PACS/topics: 75.20.En, 75.50.Kj, 75.60.Nt, 75.60.Jk

\section{Introduction}

Magnetization of ferromagnetic material is usually described by four processes: reversible and irreversible shift of domain walls, rotation of the vector magnetic polarization, and paraprocess. The area of weak magnetic fields, so-called Rayleigh region ( $R R$ ), is characteristic for the reversible motion of domain walls. In this area, the dependence of the magnetic polarization on magnetic field strength is described by the following expression:

$$
J=\mu_{0} \kappa_{P} H+\alpha H^{2},
$$

where $J$ is magnetic polarization, $H-$ magnetic field intensity, $\kappa_{p}$ - initial volume magnetic susceptibility, $\alpha$ - the Rayleigh constant and $\mu_{0}$ is the permeability of vacuum. The initial volume magnetic susceptibility $\kappa_{p}$ and the Rayleigh constant $\alpha$ are constants dependent on the type of ferromagnetic material [1].

The reason of irreversible shift of domain walls in crystalline materials outside RR is the presence of structural defects or non-ferromagnetic regions. In amorphous ferromagnetics, the role of disorders is played by mechanical stress introduced in the alloy during its preparation, as well as mechanical stress around atomic clusters. The presence of defect in the domain wall will reduce its energy and thus the domain wall will bind to the defect. The more the size of this defect approaches the thickness of the domain wall, the more the domain wall motion is braked. Magnetic reversal in RR is carried out by reversible shift of the domain walls which are not bound to inclusions. It will be interesting to know to what extent the processes in RR can be affected by different stress conditions in amorphous material. Heat treatment is frequently used for the study of mechanical stresses and their possible changes in amorphous materials. Disadvantage of this method is that simultaneously with reduction of in-

\footnotetext{
*corresponding author; e-mail: jkovac@saske.sk
}

ternal stresses atomic clusters are created [2, 3]. Thus we decided to use a heating pulse, assuming that due to the short duration of heating, clusterization will be very small.

\section{Experimental}

The FINEMET-type samples with chemical composition of $\left(\mathrm{Fe}_{73.5} \mathrm{Cu}_{1} \mathrm{Nb}_{3} \mathrm{Si}_{13.5} \mathrm{~B}_{9}\right)$ were used for the study. The first one was in as-supplied state (amorphous), the second one was annealed using one pulse heating, the third one was annealed using 20 pulse heating. Due to comparison the parameters of this sample were measured also after heat treatment at $520^{\circ} \mathrm{C}$ for $1 \mathrm{~h}$, i.e. in the nanocrystalline state. The parameters of one pulse were following: duration of pulse $100 \mathrm{~ms}$, the reached temperature $345^{\circ} \mathrm{C}$ and supplied energy $32.20 \mathrm{~J}$. Magnetic parameters of the samples were determined using magnetometer measuring stray fields of the sample, apparatus for pulse heating was placed in magnetometer [4].

First, the initial magnetic polarization curve was measured (virgin curve) and hysteresis loop in magnetic field with magnetic field strength from $-15 \mathrm{kA} / \mathrm{m}$ to $+15 \mathrm{kA} / \mathrm{m}$ (so-called large loop), then the initial magnetic polarization curve was measured in detail and hysteresis loop in magnetic field with magnetic field strength from $-100 \mathrm{~A} / \mathrm{m}$ to $+100 \mathrm{~A} / \mathrm{m}$ (detailed loop). Subsequently, pulse heating was applied, and after $20 \mathrm{~min}$ all mentioned magnetic measurements were carried out again. This process was repeated 20 times. From the large loop the following magnetic parameters were determined - magnetic polarization of saturation, measured in magnetic field of $15 \mathrm{kA} / \mathrm{m}\left(J_{s}\right)$, coercivity $\left(H_{c}\right)$ and the total magnetic anisotropy $(K)$, determined from the area above the curve of the initial magnetic polarization. The values of these parameters and the Curie temperature $\left(T_{\mathrm{C}}\right)$ for all studied samples are listed in Table I.

The course of coercivity and total magnetic anisotropy during 20 pulse heatings of amorphous samples are shown in Fig. 1. The course of detailed hysteresis loop of the studied sample is shown in Fig. 2. 
TABLE I

Selected magnetic parameters of studied samples.

\begin{tabular}{c|c|c|c|c}
\hline \hline FINEMET & $J_{s}[\mathrm{~T}]$ & $H_{\mathrm{C}}[\mathrm{A} / \mathrm{m}]$ & $\mathrm{K}\left[\mathrm{J} / \mathrm{m}^{3}\right]$ & $T_{\mathrm{C}}[\mathrm{K}]$ \\
\hline \hline amorphous & 1.139 & 14.6 & 1120 & 620 \\
\hline after 1. impulse & 1.151 & 5.78 & 618 & 620 \\
\hline after 20. impulse & 1.154 & 3.64 & 615 & 620 \\
\hline nanocryst. & 1.151 & 3.02 & 256 & 860
\end{tabular}

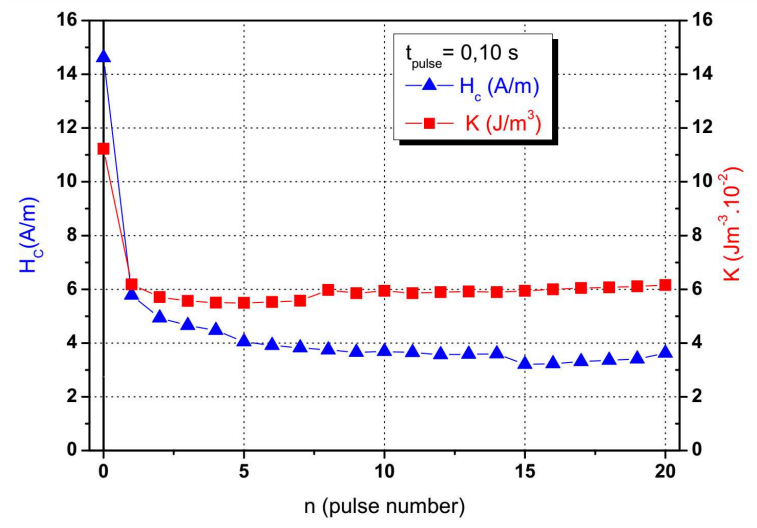

Fig. 1. The course of coercivity and total magnetic anisotropy during pulse heating of FINEMET sample.

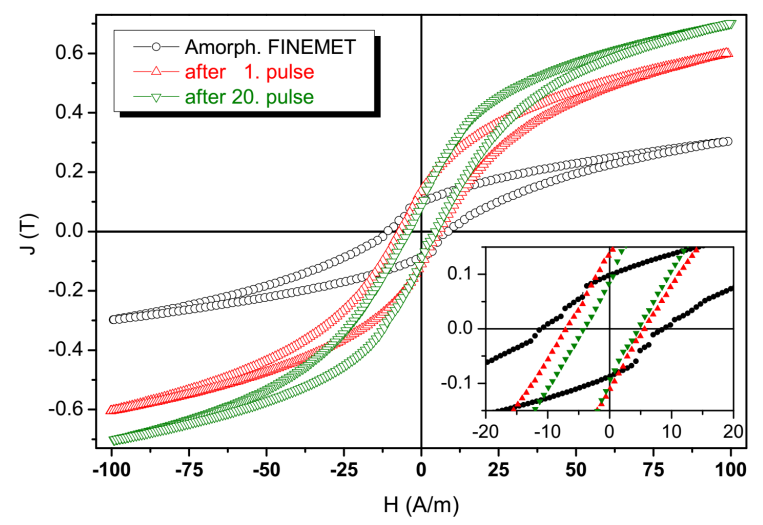

Fig. 2. Hysteresis loops of the samples of FINEMET.

\section{Results and discussion}

Initial parts of virgin curves of the samples were measured in detail. The Rayleigh parabola was fitted to the first points using the formula (1), from which $\kappa_{p}$ and $\alpha$ were determined, their values are listed in Table II. The initial parts of virgin curves of the samples with the Rayleigh parabola are shown in Fig. 3. The size of areas in which the Rayleigh parabola coincides with the initial area of the virgin curve, i.e. $\mathrm{RR}$ is for all samples listed in Table II. Relatively little difference in the size of RR is unexpected due to the large difference in coercivity of the studied samples.

There is conspicuous difference in the size of the parts of the samples magnetized within the RR, i.e. in the size of the part of samples that were magnetized by reversible displacement of domain walls. If we compare magnetic

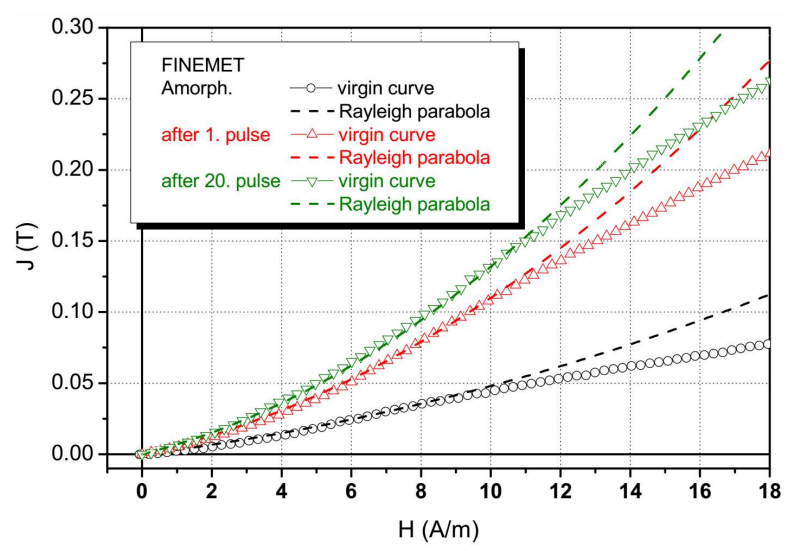

Fig. 3. The initial part of the virgin magnetic polarization curves and Rayleigh parabolas for FINEMET samples which underwent different pulse heat treatments.

polarization achieved within $\mathrm{RR}$ with saturation magnetic polarization (in the field of $15 \mathrm{kA} / \mathrm{m}$ ), we see that in the amorphous sample $2.1 \%$ of sample volume was magnetized, in the samples after pulse heating it is about $9 \%$ and in nanocrystalline sample it is $11.4 \%$ (Table II). Based on this, the mobility of domain walls in RR will significantly increase, once the pulse heating is applied. Such changes may cause decrease in the amount of structural defects in our case clusters or their reduction. But, such change caused by pulse heating is unrealistic. For this reason we will try to find out whether the increase in the thickness of the domain wall could be the possible cause of this effect. The thickness of $180^{\circ}$ domain wall can be estimated using formula $[1,5]$ :

$$
\delta_{w}=\pi \sqrt{\frac{A}{K a}},
$$

where $A$ is so-called exchange integral for which can be written $A \doteq 0.15 k T_{\mathrm{C}}, k$ is the Boltzmann constant $\left(k=1.38 \times 10^{-23} \mathrm{~J} \mathrm{~K}^{-1}\right), T_{\mathrm{C}}$ is the Curie temperature of the sample. $a$ is the lattice constant of the crystalline material and the mean distance between the atoms of amorphous alloy. For the FeSi alloy with low Si content of its value is $a=2.86 \times 10^{-10} \mathrm{~m}$. Taking into account the composition and density of FeSi and studied samples, we used this value for both nanocrystalline and amorphous samples.

From the facts mentioned above it results that expression (2) for amorphous materials can be modified as follows:

$$
\delta_{w} \doteq \pi \sqrt{\frac{0.15 k T_{\mathrm{C}}}{K a}} .
$$

The values of thickness of the $180^{\circ}$ domain wall for all samples were determined using (3) and they are listed in Table II. The values of other physical quantities, calculated from the measured data are also listed in Table II - initial volumetric magnetic susceptibility $\kappa_{p}$, the Rayleigh constant $\alpha$, size of $\mathrm{RR}$, the relative size of the sample magnetized within RR, i.e. the ratio of the maxi- 
mum magnetic polarization achieved in $\mathrm{RR}$ and magnetic polarization saturation samples $\left(\frac{J_{\text {Rayl }}}{J_{s}}\right)$ and a thickness of $180^{\circ}$ domain walls $\left(\delta_{w}\right)$.

TABLE I

The parameters calculated from the measured data.

\begin{tabular}{c|c|c|c|c|c}
\hline \hline FINEMET & $\frac{\kappa_{p}}{1000}$ & $\alpha\left[\frac{\mu \mathrm{Wb}}{\mathrm{A}^{2}}\right]$ & $\mathrm{RR}\left[\frac{\mathrm{A}}{\mathrm{m}}\right]$ & $\frac{J_{\text {Rayl }}}{J_{s}}$ & $\delta_{w}[\mathrm{~nm}]$ \\
\hline amorphous & 2.38 & 180 & $0-8$ & $2.61 \%$ & 147 \\
after 1. imp. & 4.37 & 560 & $0-10$ & $9.45 \%$ & 268 \\
after 20. imp. & 4.93 & 700 & $0-11$ & $9.81 \%$ & 267 \\
nanocryst. & 9.95 & 200 & $0-11.5$ & $11.39 \%$ & 491
\end{tabular}

In amorphous sample the part of the sample volume, which is magnetized in RR (Table II) is small, thus mobility of domain walls in the SR is small in comparison with other samples (in comparison with crystalline sample mobility of the domain walls is higher by one order [6]). This is supported also by a relatively high coercivity of the sample (Table I). This effect can be caused by the preparation procedure which introduces large internal mechanical stresses, leading to large magnetoelastic anisotropy. Stresses or structure being deformed by them, is an obstacle for domain wall motion. In view of the composition of the sample (it includes 5 components), it can be assumed that there is clustering of atoms, which also leads to reduced mobility of domain walls. Their thickness also contributes to low mobility of domain walls if their thickness does not differ much from the size of obstacles.

After the first pulse heating a significant decrease in internal stresses in the sample occurs, leading to a significant decrease in coercivity and mechanical stresses and thus decrease in the total anisotropy of the sample. As a consequence, the thickness of the domain wall grows approximately twice. As this heat treatment causes only slight growth of clusters, there is a significant increase in the mobility of domain walls. After the next 19 heating pulses, the sample properties changed only slightly.

For nanocrystalline samples the size of RR is comparable with that one for amorphous sample. However, the part of the sample, which is remagnetized by reversible shift of domain walls in RR (Table II) is significantly larger. This suggests to a large increase in the mobility of domain walls. Such assumption is supported by a very low coercivity with respect to the amorphous sample.

The cause is probably the process of nanocrystalline transformation, in which degradation of the majority of internal stresses occurs, thereby magnetoelastic anisotropy and also the number and size of the distorted regions reduce, as well as the number of distorted regions that brake the domain wall motion. There is also the collapse of clusters into crystallites with dimensions of about $10 \mathrm{~nm}$, whose dimensions are small in comparison with the thickness of the domain walls. Simultaneously there is also increase in the thickness of domain wall.

\section{Conclusions}

Pulse heating of amorphous sample leads to a significant decrease in internal stresses in the sample without affecting of clusterization. The consequence of the decrease of internal mechanical stress is the significant decrease in total anisotropy, since magnetoelastic anisotropy in amorphous ferromagnetics is dominant. Reduction of the energy required for the magnetization in direction of the magnetization by almost $50 \%$ and also increase in the thickness of domain walls, while maintaining the size of the obstacles to their movement, lead to a significant increase of mobility of domain walls. The consequence of the increased mobility of domain walls is that in the Rayleigh area 3 times higher portion of the sample (Table II) is magnetized. Consequently due to the increased mobility of domain walls the decrease in coercivity (Fig. 1) and also change in the hysteresis loop shape (Fig. 2) are observed.

\section{Acknowledgments}

This research was supported by VEGA grants No. $1 / 0413 / 15,1 / 0377 / 16$, and $1 / 0164 / 16$ of the Scientific Grant Agency of the Ministry for Education of the Slovak Republic and also by the project "Centre of Excellence for Integrated Research \& Exploitation of Advanced Materials and Technologies in Automotive Electronics" ITMS 26220120055.

\section{References}

[1] J.M.D. Coey, Magnetism and Magnetic Materials, Oxford University Press, 2009.

[2] L. Novák, L. Potocký, S. Uličiansky, É. Kisdi-Koszó, A. Lovas, J. Takács, R. Mlýnek, J. Magn. Magn. Mater. 26, 118 (1982).

[3] É. Kisdi-Koszó, L. Potocký, M. Hrabčák, L. Novák, A. Lovas, J. Magn. Magn. Mater. 92, 181 (1990).

[4] J. Kováč, L. Novák, L. Hubač, J. Electr. Eng. 66, $142(2015)$.

[5] S. Chikazumi, Physics of Ferromagnetism, Oxford University Press, Oxford 1997.

[6] L. Novák, J. Kováč, Acta Elektrotechn. Informat. 13, 61 (2013). 